\title{
A Combination of Wireless Multicast Advantage and Hitch-Hiking
}

\author{
My T. Thai, Yingshu Li, and Ding-Zhu Du
}

\begin{abstract}
In the minimum energy broadcast problem, each node adjusts its transmission power to minimize the total energy consumption while still delivering data to all the nodes in a network. The minimum energy broadcast problem is proved to be NP-complete. The Wireless Multicast Advantage (WMA) is that a single transmission can be received by all the nodes that are within the transmission range of a transmitting node. The Hitch-hiking model introduced recently in [1] takes advantage of the physical layer to combine partial signals containing the same data in order to decode a complete message. In this letter, we take advantage of both WMA and Hitch-hiking to design an energy-efficient broadcast tree algorithm with Hitch-hiking (BHH). The approximation ratio of $\mathrm{BHH}$ is within a factor of $O(\log n)$ where $n$ is the number of nodes in the network. The simulation results show that $\mathrm{BHH}$ reduces the total energy of the broadcast tree greatly.
\end{abstract}

Index Terms - Wireless networks, energy efficiency, broadcast algorithms, hitch-hiking.

\section{INTRODUCTION}

$\mathbf{R}$ ECENT improvements in affordable and efficient integrated electronic devices have a considerable impact on advancing the state of wireless networks in the form of ad hoc networks and sensor networks. Wireless networks are used in a wide range of applications including security, health care, military, and many other fields. A critical issue in wireless networks is power scarcity. Therefore, energy efficiency is critical in designing any algorithm for wireless networks to prolong network lifetime.

In this letter, we study the problem of minimizing the total broadcast energy in wireless networks. The broadcast problem in wireless networks is to decide a transmission power level for each node so that the source node can broadcast to all the other nodes either directly or indirectly through the relay nodes with the objective of minimizing the total energy consumption. Our focus is on source-initiated broadcast. Our objective is to construct a minimum-power broadcast tree, rooted at the source node, including all the nodes. Our key idea is to reduce the total energy consumption of the broadcast tree by taking the Hitch-hiking model [1] and Wireless Multicast Advantage (WMA) into consideration. The performance ratio of our proposed algorithm $\mathrm{BHH}$ is bounded by $O(\log n)$ where $n$ is the number of nodes in a network. The simulation

Manuscript received June 4, 2005. The associate editor coordinating the review of this letter and approving it for publication was Dr. Sarah Kate Wilson.

M. T. Thai and D.-Z. Du are with the Dept. of Computer Science and Engineering, University of Minnesota, Minneapolis, MN (e-mail: \{mythai, dzd\}@cs.umn.edu).

Y. Li is with the Dept. of Computer Science, Georgia State University, Atlanta, GA (e-mail: yli@cs.gsu.edu).

Digital Object Identifier 10.1109/LCOMM.2005.12004 results also show that the total power of the broadcast tree constructed using $\mathrm{BHH}$ is almost $77 \%$ less than that of Broadcast Increment Power (BIP) [2], which is a well known algorithm for minimizing the total energy consumption of a broadcast tree.

\section{Preliminaries}

\section{A. Communication Model}

We assume that any node in the network can be used as a relay node to forward data to other nodes in the network. All nodes are equipped with omnidirectional antennas so that if node $i$ transmits data to node $j$, all nodes closer to $i$ than $j$ will also receive the transmitted data. We also assume that all nodes can adjust their power levels. Each node can choose its transmission power from 0 to some maximum value $p_{\max }$.

Attenuation model commonly assumes that signal power falls proportional to $d^{\alpha}$, where $d$ is the signal traveling distance, and $\alpha$ is an environmentally dependent real constant between 2 and 4 [5]. A node $j$ can properly receive a signal from a node $i$ which has a power level $p_{i}$ if $p_{i} \geq \gamma d_{i j}^{\alpha}$, where $\gamma$ represents the receiver's power threshold for signal detection or Signal to Noise Ratio (SNR), often normalized to 1. Hence, for node $i$ in the network, the power required to successfully transmit data to node $j$ is given by: $p_{i j} \geq d_{i j}^{\alpha}$.

\section{B. Hitch-Hiking Model}

In [1], the authors introduced two thresholds on the SNR: $\gamma_{p}$ - threshold needed for successfully decoding the packet payload and $\gamma_{a c q}$ - the threshold required for a successful time acquisition.

A packet received with a SNR $\gamma$ is: (1) Full reception if $\gamma_{a c q}<\gamma_{p} \leq \gamma$, (2) Partial reception if $\gamma_{a c q} \leq \gamma<\gamma_{p}$, and (3) Failed reception if $\gamma<\gamma_{a c q}$.

The basic idea of the Hitch-hiking is that if a node receives the packet containing the same information $k$ times from different neighbors with $\gamma_{i}$ such as $\sum_{i=1}^{k} \gamma_{i} \geq \gamma_{p}$ and $\gamma_{i} \geq$ $\gamma_{a c q}$ for all $i$, then that node can successfully receive the packet. In designing the solution, the authors assumed that $\gamma_{p}=1$ and $\gamma_{a c q}=0$ for simplicity.

\section{Network Model}

Let $V$ denote the collection of wireless nodes and let $G=$ $(V, E)$ denote the directed graph on $V$ that contains all edges when each node transmits at its maximum transmission power. Every node $i \in V$ has an associated transmission power level $p_{i}$. When node $i$ transmits a packet, the amount of reception 
by node $j$ is quantified by the coverage of node $j$ defined as $c\left(\frac{\gamma_{i j}}{\gamma_{p}}\right)$ where $c(\beta)$ is the coverage function given by:

$$
c(\beta)= \begin{cases}1 & \text { if } \beta \geq 1 \\ \beta & \text { if } \frac{\gamma a c q}{\gamma_{p}}<\beta<1 \\ 0 & \text { otherwise }\end{cases}
$$

Thus, the coverage provided by node $i$ on node $j$ is:

$$
c(i j)= \begin{cases}1 & \text { if } \frac{p_{i}}{d_{i j}^{\alpha}} \geq \gamma_{p} \\ \frac{p_{i}}{d_{i j}^{\alpha}} & \text { if } \gamma_{a c q} \leq \frac{p_{i}}{d_{i j}^{\alpha}}<\gamma_{p} \\ 0 & \text { otherwise }\end{cases}
$$

Let a directed graph $T=\left(V, E^{\prime}\right)$ represent the broadcast tree where $V$ is the set of all the nodes in $G$ and $E^{\prime}$ is the set of all the edges corresponding to the communication links between nodes after the power level is set. For each directed edge $i j \in E^{\prime}$, the coverage provided by an edge $i j$ to the node $j$ is calculated by (1).

\section{RELATED WORK}

The problem of minimum-energy broadcast in wireless networks has received significant attention over the last few years. Liang [3] proved that this problem is NP-complete. For an extensive discussion of minimum-energy broadcast tree algorithms, please refer to [4]. In this section, we briefly review the work most relevant to our approach.

Broadcast Increment Power (BIP) [2] is a popular heuristic to construct a minimum-energy broadcast tree rooted at the source node. Starting at the source node, BIP adds one node at a time to the broadcast tree where each added node is an uncovered node which can be added to the tree at the minimum additional cost. Note that BIP just adds one node at a time, thus failing to fully exploit the advantage of WMA.

In [1], the authors introduced the Wireless Multicast with Hitch-hiking (WMH) algorithm. First, WMH constructs the Minimum Spanning Tree (MST) rooted at the source node. Starting from the source node, at each step, the algorithm chooses a fully covered node $u$ and sets the power level of node $u$ to the power needed to reach its furthest children and grandchildren in the MST while obtaining the maximum power reduction in $u$ 's local region. Note that WMH constructs the broadcast tree based on the MST and adjusts the power level of each node based on local optimization. It does not take full advantages of WMA and Hitch-hiking.

In the following section, we describe our algorithm that at each step, the algorithm adds a set of nodes into the tree to further exploit the properties of WMA. It also considers the partial signal that a node can obtain from other nodes, not only from its parent and grandparent.

\section{The Broadcast with Hitch-Hiking Algorithm}

Let us first introduce the following notations:

- $c_{h}(i j)$ : the coverage of node $j$ provided by node $i$ at the iteration $h . c(i j)$ is defined in formula (1)

- $c_{h}(j)$ : the total coverage of node $j$ provided by all the other nodes at the iteration $h . c(j)=\sum_{i \in V} c(i j)$

- $p_{h}(i)$ : the power level of node $i$ at the iteration $h$

- $N(i)$ : the neighbors of node $i$
Let $P_{h}$ be the set of nodes that are not fully covered in the network at the iteration $h$. Thus, $F_{h}=V-P_{h}$ is the set of nodes that are fully covered in the network at the iteration $h$. In the algorithm, sometimes we refer the set of fully covered nodes and the set of partially covered nodes as $F$ and $P$ respectively if we do not specify the iteration step. Let us first define a benefit ratio $r(i)$ :

$$
r(i)=\frac{p_{h}(i)-p_{h-1}(i)}{\left|F_{h}-F_{h-1}\right|}
$$

Note that $p_{h}(i)-p_{h-1}(i)$ is the incremental power of node $i$ at the iteration $h$ and the denominator of $r(i)$ is the number of new fully covered nodes after increasing the power level of node $i$. Thus, $\left|F_{h}-F_{h-1}\right|=\left|P_{h-1}-P_{h}\right|=\mid\{j \mid j \in$ $\left.P_{h-1} \& c_{h-1}(j)+c_{h}(i j) \geq 1\right\} \mid$.

In order to minimize the total power consumption, we not only want the incremental power of a node at each step to be as small as possible, but also want the number of fully covered nodes at each iteration to be as large as possible. This leads to the value $r(i)$ must be the minimum one at each iteration. If ties occur, we choose the node that has the minimum value $r^{\prime}(i)$ where

$$
r^{\prime}(i)=\frac{p_{h}(i)-p_{h-1}(i)}{\sum_{j \in P_{h-1}}\left(\min \left\{1,\left(c_{h-1}(j)+c_{h}(i j)\right)\right\}\right)} .
$$

The denominator of $r^{\prime}(i)$ is the sum of the updated coverage of all nodes $j$ after increasing the power level of node $i$ where $j$ is in the partially covered node set of the previous iteration, $P_{h-1}$. Now, we are ready to introduce BHH.

\section{Algorithm Description:}

The BHH algorithm starts at the source node $S$. The initial coverage of $S$ is $c_{0}(S)=1$ and the initial coverage $c_{0}(i)=0$ for all $i \in V$ and $i \neq S$. Therefore, $F_{0}=\{S\}$ and $P_{0}=$ $V-F_{0}$. Let $T=(V, \emptyset)$ be the initial broadcast tree. First, adjust the power level of node $S, p(S)$ to cover each node $i$ where $i \in N(S)$ and $i \in P_{0}$. At each power level, the tuple $\left\langle r(S), r^{\prime}(S)\right\rangle$ is calculated. The power level of node $S$ is set to the level at which $\left\langle r(S), r^{\prime}(S)\right\rangle$ is the minimum one. We then update the coverage $c(k)$ where $k \in P_{0}$ and $k$ can fully or partially receive a signal from node $S$, and add all the directed edges from node $S$ to other nodes $k$ with the associated coverage to $T$. If $c(k) \geq 1$, remove $k$ from the partially covered node set $P$ and add it to the fully covered node set $F$. For all the nodes in $F$, we iteratively repeat the process until $P=\emptyset$, i.e, all the nodes in $T$ are fully covered. The algorithm is shown in Algorithm 1.

Theorem 1: Given a network of size $n$, the approximation ratio of BHH is within a factor of $O(\log n)$

Proof: Due to space limitation, detailed proof is omitted in this letter. Let $C$ and $C^{*}$ denote the total power assumption of the broadcast tree obtained from $\mathrm{BHH}$ and the optimal one respectively. Using the similar technique in [7], we have $C \leq$ $2 C^{*} \cdot H(n)$ where $H(n)$ denotes the $n^{t h}$ harmonic number. Since $H(n) \leq 1+\ln n$, we have $C \leq C^{*} \cdot O(\log n)$.

\section{Simulation Results}

In this section, we evaluate the performance of BHH by comparing it to two algorithms, BIP and WMH. Since BIP 


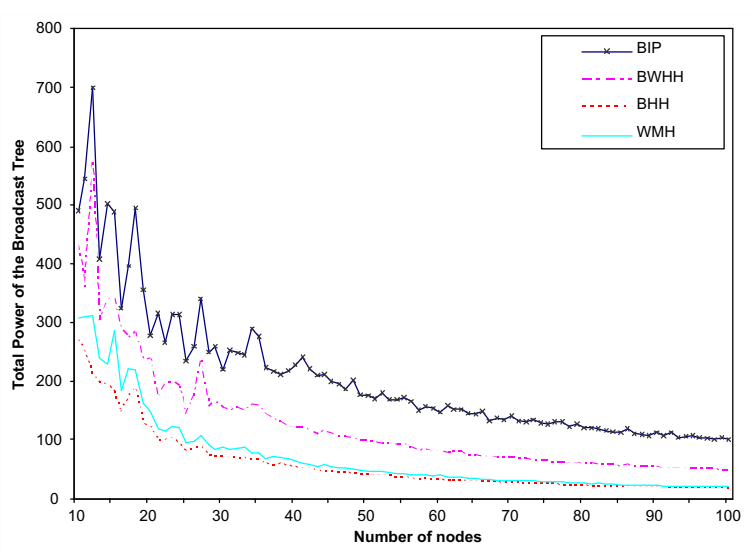

(a) Total Power Cost

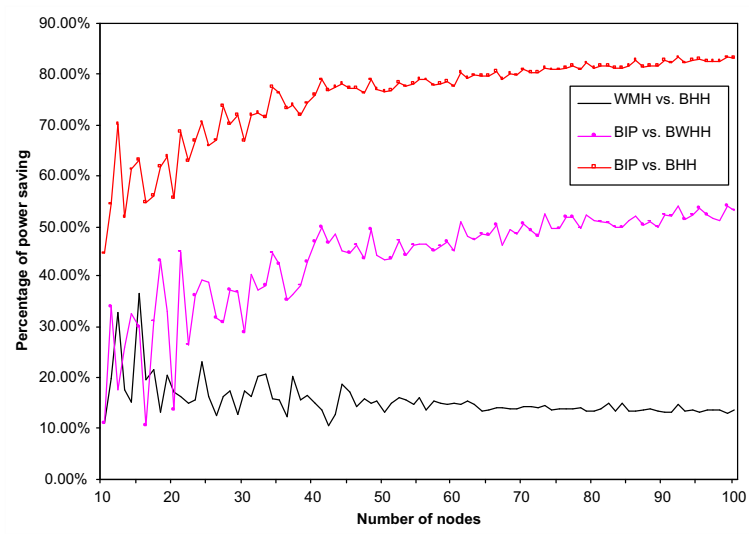

(b) The improvement of BHH

Fig. 1. A comparison of total power, $\alpha=2$.

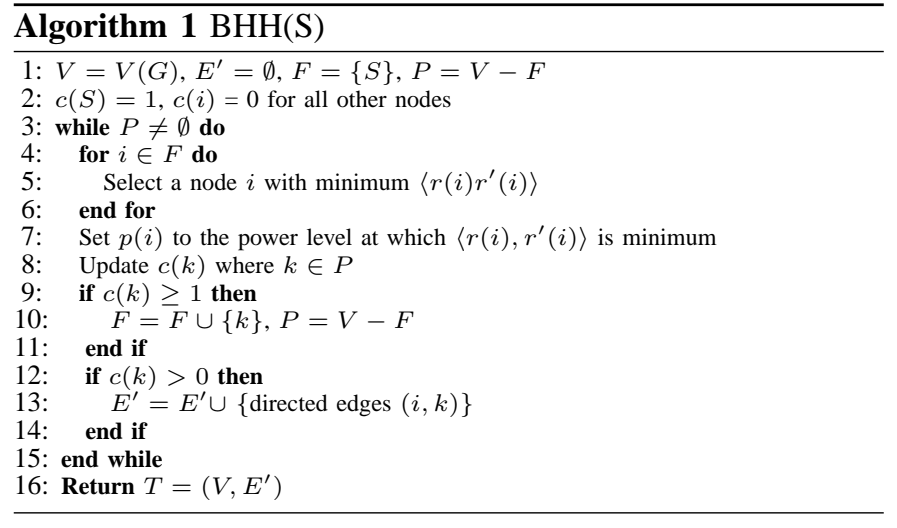

does not use Hitch-hiking model, it is also our interest to compare our algorithm without using Hitch-hiking (BWHH) to BIP. The BWHH algorithm can be obtained from $\mathrm{BHH}$ with a change on the coverage function. Instead of using the formula (1), the coverage provided by node $i$ on node $j$ is defined as:

$$
c(i j)= \begin{cases}1 & \text { if } \frac{p_{i}}{d_{i j}^{\alpha}} \geq \gamma_{p} \\ 0 & \text { otherwise }\end{cases}
$$

In these simulations, we simulated a stationary network randomly distributed over the same deployment region. The number of nodes $n$ increased from 10 to 100 . The maximum power level of each node was randomly assigned on each simulation setup. We ran 100 simulations for each value of $n$. In all simulations, a fixed value $\alpha=2$ was used. The performance metric is the average of the total power of the broadcast tree obtained from each algorithm.

Fig. 1(a) illustrates the performance of the proposed algorithm $\mathrm{BHH}$ compared to BIP and WMH. As can be seen, the total power of the broadcast tree constructed using $\mathrm{BHH}$ is almost $77 \%$ less than that of BIP, and $15 \%$ less than that of WMH. Also, the total power of a broadcast tree decreases when the number of nodes increases. This is because as the network density increases, more nodes are available to work as relay nodes and the nodes are becoming closer. Also, as revealed in Fig. 1(a), the power of the broadcast tree obtained from BWHH is $49 \%$ less than that of BIP. This indicates that our algorithm takes more advantage of WMA than BIP does.

In Fig. 1(b), we present the comparison of the improvement on saving energy over the other three algorithms in percentage: BIP vs. BHH, BIP vs. BWHH, BIP vs. WMH. Fig. 1(b) shows that $\mathrm{BHH}$ is the one that can save the most energy compared to BIP, WMH, and BWHH. This analysis indicates that combining WMA and Hitch-hiking model does achieve a better result.

\section{CONCLUSION}

In this letter, we studied the minimum-energy broadcast problem, which is proved to be NP-complete. We proposed the $\mathrm{BHH}$ algorithm based on the Hitch-hiking model. This algorithm takes advantage of WMA and of the Hitch-hiking concept. The simulation results showed that $\mathrm{BHH}$ can reduce the total power of the constructed broadcast tree by as much as $77 \%$ compared to BIP, and $15 \%$ compared to WMH. We also compared our algorithm without using Hitch-hiking to BIP, the total power of the broadcast tree can be reduced by $49 \%$. We also proved that the approximation ratio of our proposed algorithm is within a factor of $O(\log n)$.

\section{REFERENCES}

[1] M. Agarwal, J. H. Cho, L. Gao, and J. Wu, "Energy efficient broadcast in wireless ad hoc networks with hitch-hiking," in Proc. IEEE INFOCOM 2004, pp. 2097-2108.

[2] J. E. Weiselthier, G. D. Nguyen, and A. Ephremides, "On the construction of energy-efficient broadcast and multicast trees in wireless networks," in Proc. IEEE INFOCOM 2001, pp. 585-594.

[3] W. Liang, "Constructing minimum-energy broadcast trees in wireless ad Hoc networks," in Proc. IEEE MOBIHOC 2002, pp. 112-122.

[4] F. Ingelrest, D. Simplot-Ryl, and I. Stojmenovic, "Energy-efficient broadcasting in wireless mobile ad hoc networks," in Resource Management in Wireless Networking, M. Cardei, I. Cardei, and D.-Z. Du (eds.). Kluwer Academic Publishers, 2004.

[5] T. S. Rappaport, Wireless Communications: Principles and Practice. Prentice Hall, 1996.

[6] J. Cartigny, D. Simplot, and I. Stojmenovic, "Localized minimum-energy broadcasting in ad-hoc networks," in Proc. IEEE INFOCOM 2003, pp. 2210-2217.

[7] F. Bian, A. Goel, C. S. Raghavendra, and X. Li, "Energy-efficient broadcasting in wireless ad hoc networks: lower bounds and algorithms," J. Interconnection Networks, vol. 3, pp. 149-166, Dec. 2002. 